MATHEMATICS OF COMPUTATION

Volume 70, Number 235, Pages 1089-1111

S 0025-5718(00)01288-6

Article electronically published on October 16, 2000

\title{
HERMITE INTERPOLATION BY PYTHAGOREAN HODOGRAPH CURVES OF DEGREE SEVEN
}

\author{
B. JÜTTLER
}

\begin{abstract}
Polynomial Pythagorean hodograph (PH) curves form a remarkable subclass of polynomial parametric curves; they are distinguished by having a polynomial arc length function and rational offsets (parallel curves). Many related references can be found in the article by Farouki and Neff on $C^{1}$ Hermite interpolation with $\mathrm{PH}$ quintics. We extend the $C^{1}$ Hermite interpolation scheme by taking additional curvature information at the segment boundaries into account. As a result we obtain a new construction of curvature continuous polynomial PH spline curves. We discuss Hermite interpolation of $G^{2}\left[C^{1}\right]$ boundary data (points, first derivatives, and curvatures) with $\mathrm{PH}$ curves of degree 7. It is shown that up to eight possible solutions can be found by computing the roots of two quartic polynomials. With the help of the canonical Taylor expansion of planar curves, we analyze the existence and shape of the solutions. More precisely, for Hermite data which are taken from an analytical curve, we study the behaviour of the solutions for decreasing stepsize $\Delta$. It is shown that a regular solution is guaranteed to exist for sufficiently small stepsize $\Delta$, provided that certain technical assumptions are satisfied. Moreover, this solution matches the shape of the original curve; the approximation order is 6 . As a consequence, any given curve, which is assumed to be $G^{2}$ (curvature continuous) and to consist of analytical segments can approximately be converted into polynomial $\mathrm{PH}$ form. The latter assumption is automatically satisfied by the standard curve representations of Computer Aided Geometric Design, such as Bézier or B-spline curves. The conversion procedure acts locally, without any need for solving a global system of equations. It produces $G^{2}$ polynomial PH spline curves of degree 7 .
\end{abstract}

\section{INTRODUCTION}

Polynomial parametric curves $\mathbf{x}(t)=\left\{x_{1}(t), x_{2}(t)\right\}$ (such as Bézier or B-spline curves) with polynomial components $x_{i}(t)$ form the mathematical foundation of the various computer-aided design tools that are currently in use for the design and manufacturing of planar shapes (see [5 [10]). For instance, a polynomial Bézier curve of degree $n$ is given by the parametric representation

$$
\mathbf{x}(t)=\left\{x_{1}(t), x_{2}(t)\right\}=\sum_{i=0}^{n} B_{i}^{n}(t) \mathbf{b}_{i}, \quad t \in[0,1],
$$

Received by the editor February 25, 1999 and, in revised form, August 12, 1999.

2000 Mathematics Subject Classification. Primary 68U07; Secondary 53A04, 65D17.

Key words and phrases. Hermite interpolation, Pythagorean hodograph curves, computer aided geometric design. 
with control points $\mathbf{b}_{i}=\left\{b_{i, 1}, b_{i, 2}\right\} \in \mathbb{R}^{2}$, where the $B_{i}^{n}(t)=\left(\begin{array}{c}n \\ i\end{array}\right) t^{i}(1-t)^{n-i}$ are the Bernstein polynomials of degree $n$. The Pythagorean hodograph $(\mathrm{PH})$ curves are an interesting subclass of these curves; they are distinguished by the fact that the components of the first derivative vector $\mathbf{x}^{\prime}(t)=\left\{x_{1}^{\prime}(t), x_{2}^{\prime}(t)\right\}$ (also sometimes called the hodograph) satisfy the Pythagorean condition

$$
x_{1}^{\prime 2}+x_{2}^{\prime 2}=\sigma^{2}
$$

for some polynomial $\sigma=\sigma(t)$. That is, the components of the first derivative vector (or hodograph) belong to a Pythagorean triple in the polynomial ring $\mathbb{R}[t]$. Clearly, $\sigma$ is then a polynomial of degree $n-1$.

Pythagorean hodograph curves have been introduced and studied by Farouki and various coauthors in a number of remarkable publications (see [6] and the references cited therein). The Pythagorean condition entails a number of useful properties:

- The arc length of any segment $t \in\left[t_{0}, t_{1}\right]$ can be computed exactly, without numerical quadratures:

$$
S=\int_{t_{0}}^{t_{1}} \sqrt{x_{1}^{\prime 2}+x_{2}^{\prime 2}} d t=\int_{t_{0}}^{t_{1}}|\sigma(t)| d t .
$$

(Note that sign changes of $\sigma$ correspond to degenerate points (cusps) of the $\mathrm{PH}$ curve, which have to be avoided in applications. The interpolation scheme described below is guaranteed to give regular solutions.) This property is especially advantageous in applications such as NC milling or robotics, where the tool has to be moved along a trajectory with a certain specified speed or "feedrate" (cf. [8, 9]).

- The offsets (or parallel curves) of Pythagorean hodograph curves can be represented exactly by rational parametric curves. Offset curves and their approximation have attracted a great deal of research from the geometric design community, as they are useful in numerous applications. See the excellent survey by Elber et al. for references and a comparison of various approximation techniques [4].

Using PH curves instead of "ordinary" polynomial curves, however, it is possible to bypass the need for an approximation. The offset (or parallel curve) of a given $\mathrm{PH}$ curve (1.1) at a certain distance $d$ is given by

$$
\mathbf{x}_{d}=\mathbf{x}+\frac{d}{\sigma}\left\{-x_{2}^{\prime}, x_{1}^{\prime}\right\}=\frac{1}{\sigma}\left\{\sigma x_{1}-d x_{2}^{\prime}, \sigma x_{2}+d x_{1}^{\prime}\right\},
$$

hence it can be represented exactly as a rational Bézier curve of degree $2 n-1$.

A number of methods are available that deal with the construction of tangent continuous $\left(C^{1}\right.$ or $\left.G^{1}\right)$ piecewise Pythagorean hodograph curves, mainly via Hermite interpolation. Farouki and Neff [6] solve the $C^{1}$ Hermite interpolation problem with the help of PH quintics. Meek and Walton 13 use PH cubics (also called Tschirnhausen cubics) for matching point and tangent (i.e., $G^{1}$ ) boundary data. Moreover it is shown that if the Hermite data stem from a sufficiently smooth function, then the approximation order of the Tschirnhausen cubic is four.

For many applications it is advantageous to use spline curves with continuous curvature function (i.e., $G^{2}$ curves). For instance, in NC milling applications, using such curves produces motions with continuous accelerations, hence continuous cutting forces. This will increase the possible speed of the manufacturing process, and/or the lifetime of the machinery. 
The construction of $C^{2} \mathrm{PH}$ interpolating splines has been discussed by Albrecht and Farouki [1]. The solution process involves solving a global system of quadratic equations with the help of the homotopy method. Clearly, the use of a global nonlinear system restricts the number of data that possibly can be handled with this approach. Even if the size of the nonlinear system increases only linearly with the number $n$ of data, the number of solutions (counted with multiplicities over the projective domain, see [1) equals $2^{n}$ !

Walton and Meek [17. use cubic and quintic polynomial PH spirals for interactively designing $G^{2}$ parametric curves.

Pottmann [16] uses rational PH curves of degree 6 for Hermite interpolation of $G^{2}$ boundary data. His approach is based on the so-called dual representation of a curve, as the envelope of its tangents. Unlike polynomial PH curves, however, their rational counterparts no longer have a simple arc-length function. Nevertheless, the offset curves are still rational.

The present paper discusses the construction of a curvature continuous polynomial PH spline curve via Hermite interpolation of $G^{2}\left[C^{1}\right]$ boundary data (points, first derivatives, and curvatures) by $\mathrm{PH}$ curves of degree 7 . As an advantageous feature of the construction presented, it is possible to convert any given planar $G^{2}$ curve approximately into polynomial Pythagorean hodograph form without solving a global nonlinear system. The conversion procedure acts locally, as it is based on Hermite interpolation.

There are various reasons for choosing $G^{2}\left[C^{1}\right]$ boundary data, rather than just tangent and curvature information.

- In many applications it is necessary to approximate both the original curve and its parameterization, as the latter may represent a desired speed distribution along the curve. Also, using purely geometric curve constructions (without taking the information about the first derivatives into account) may produce poorly parameterized curves.

- Using the information about the length of the first derivatives also offers some computational advantages: the interpolating curve can be found by solving a single equation of degree 4 . Consequently, criteria for the existence of solutions are available. Alternatively one might consider using $\mathrm{PH}$ quintics in order to match $G^{2}$ boundary data. The latter approach, however, would produce a highly nonlinear system of equations that could only be dealt with numerically.

The remainder of this paper is organized as follows. The next section summarizes some basic facts about $\mathrm{PH}$ curves and their construction with the help of stereographic projection. Section 3 discusses the problem of $G^{2}\left[C^{1}\right]$ Hermite interpolation by $\mathrm{PH}$ curves of degree 7 . We derive a procedure for computing the solutions. Section 4 is devoted to the asymptotic behaviour of both solutions and solvability, by discussing the following question: Provided that the $C^{2}\left[G^{1}\right]$ boundary data stem from an analytical curve, how does the solution to the interpolation problem depend on the distance between the segment end points? Finally, Section 5 formulates an approximation procedure for converting arbitrary planar curves into globally curvature continuous (i.e., $G^{2}$ ) polynomial $\mathrm{PH}$ spline curves of degree 7 . 


\section{Pythagorean hodograph CURVES}

Pythagorean hodograph $(\mathrm{PH})$ curves can be constructed with the help of the following observation which is based on an algebraic result concerning Pythagorean triples in unique factorization domains. Note that we are using a slightly different formulation than Farouki and Neff [6] Definition 1], in order to derive a geometric interpretation of the formula.

Lemma 2.1. A polynomial Bézier curve $\mathbf{x}=\mathbf{x}(t)$ of degree $n$ is a PH curve if and only if its first derivative vector (hodograph) $\mathbf{x}^{\prime}=\mathbf{x}^{\prime}(t)$ can be expressed in the form

$$
\left\{x_{1}^{\prime}, x_{2}^{\prime}\right\}=\left\{2 w u_{1}\left(1+u_{2}\right), w\left(\left(1+u_{2}\right)^{2}-u_{1}^{2}\right)\right\}
$$

with real polynomials $w(t), u_{1}(t)$, and $u_{2}(t)$ of certain degrees $k, m$, and $m$, respectively, where $n=2 m+k+1$. The associated polynomial $\sigma(t)$ satisfying the Pythagorean condition $\sigma^{2}=x_{1}^{\prime 2}+x_{2}^{\prime 2}$ is then given by

$$
\sigma=w\left(u_{1}^{2}+\left(1+u_{2}\right)^{2}\right) .
$$

Proof. According to a result on Pythagorean triples in unique factorization domains, any solution of (1.2) can be represented as

$$
\left\{x_{1}^{\prime}, x_{2}^{\prime}\right\}=\left\{w\left(v_{1}^{2}-v_{2}^{2}\right), 2 w v_{1} v_{2}\right\} \quad \text { and } \quad \sigma=w\left(v_{1}^{2}+v_{2}^{2}\right),
$$

with real polynomials $w(t), v_{1}(t), v_{2}(t)$ of degrees $k, m$ and $m$, respectively, where $n=2 m+k+1$ (see [6] 12]). The formulas (2.1) and (2.3) are equivalent. This can be seen by substituting $u_{1}=\left(v_{1}-v_{2}\right) / \sqrt{2}, u_{2}=\left(v_{1}+v_{2}\right) / \sqrt{2}-1$ in (2.1), and conversely $v_{2}=\left(u_{2}+1-u_{1}\right) / \sqrt{2}, v_{1}=\left(u_{1}+u_{2}+1\right) / \sqrt{2}$ in (2.3).

After choosing the hodograph, the corresponding $\mathrm{PH}$ curve can easily be computed by integrating the components of the hodograph. The integration constants specify the starting point $\mathbf{x}(0)$ of the curve.

Remark 2.2. In the remainder of this paper we will assume that the polynomial $w(t)$ has strictly positive values in $[0,1]$. Zeros of this polynomial within $[0,1]$ would cause the resulting PH curve to have a singularity; therefore, they are excluded. Without loss of generality one may then choose the polynomial $w$ to be positive.

Lemma 2.1 admits a geometric interpretation:

Lemma 2.3. Consider the unit tangent of the PH curve (1.1) which has been obtained from (2.1),

$$
\overrightarrow{\mathbf{t}}(t)=\frac{1}{\sigma}\left\{x_{1}^{\prime}(t), x_{2}^{\prime}(t)\right\}=\left\{\frac{2 u_{1}\left(1+u_{2}\right)}{u_{1}^{2}+\left(1+u_{2}\right)^{2}}, \frac{\left(1+u_{2}\right)^{2}-u_{1}^{2}}{u_{1}^{2}+\left(1+u_{2}\right)^{2}}\right\} .
$$

For $t \in[0,1]$, the unit tangent $\overrightarrow{\mathbf{t}}(t)$ traces a segment of the unit circle. For any parameter value $t \in \mathbb{R}$, the three points $\mathbf{z}=\{0,-1\}, \overrightarrow{\mathbf{t}}(t)$, and $\mathbf{u}(t)=\left\{u_{1}(t), u_{2}(t)\right\}$ are collinear.

Proof. The unit tangent can be rewritten as $\alpha(t) \mathbf{u}(t)+\beta(t) \mathbf{z}$ with the rational functions $\alpha=2\left(1+u_{2}\right) /\left(\left(1+u_{2}\right)^{2}+u_{1}^{2}\right)$ and $\beta=1-\alpha$.

Thus, the unit tangent $\overrightarrow{\mathbf{t}}(t)$ is obtained by stereographic projection of the curve $\mathbf{u}(t)$ onto the unit circle, where the centre of projection is chosen at $\mathbf{z}=\{0,-1\}$. See Figure $1 \mathrm{a}$ for an illustration. The curve $\mathbf{u}(t)$ will be referred to as the preimage curve. 


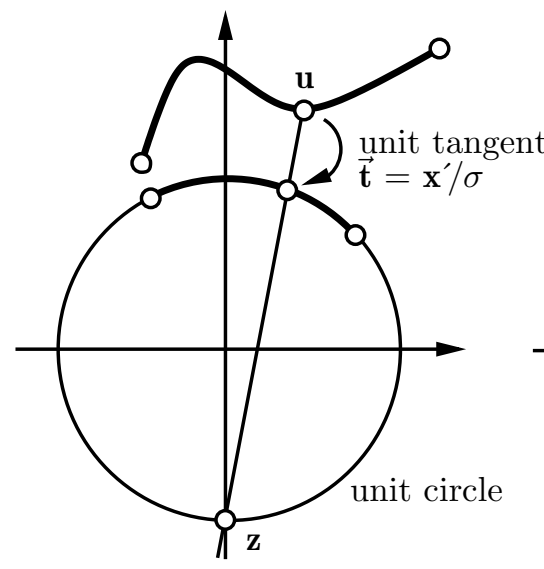

(a)

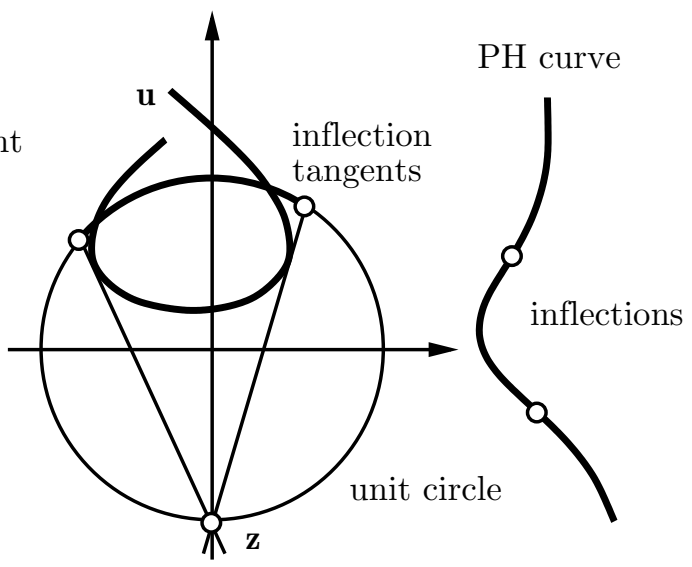

(b)

Figure 1. (a) Geometric interpretation of Lemma 2.1 as a stereographic projection. (b) Inflection of the $\mathrm{PH}$ curve corresponds to tangents of $\mathbf{u}$ passing through $\mathbf{z}$.

Remark 2.4. The hodograph formula 2.1 has a symmetry: the preimage curves $\mathbf{u}(t)=\left\{u_{1}(t), u_{2}(t)\right\}$ and $2 \mathbf{z}-\mathbf{u}(t)=\left\{-u_{1}(t),-2-u_{2}(t)\right\}$ produce the same $\mathrm{PH}$ curves. Both curves are centrally symmetric with respect to $\mathbf{z}=\{0,-1\}$.

From the geometric interpretation we conclude some relations between the geometric properties of the preimage curve $\mathbf{u}$ and the resulting $\mathrm{PH}$ curve $\mathbf{x}(t)$.

- The PH curve has a singular point at $t=t_{0}$ (i.e., $\left.\mathbf{x}^{\prime}\left(t_{0}\right)=\{0,0\}\right)$ iff $t_{0}$ is a zero of $w(t)$ (but this was excluded in Remark 2.2 for $\left.t_{0} \in[0,1]\right)$ or $\mathbf{u}\left(t_{0}\right)=\mathbf{z}$, i.e., iff the preimage curve passes through the centre of projection.

- The PH curve has vanishing curvature at $t=t_{0}$ (inflection or flat point of higher order) iff the tangent of the preimage curve $\mathbf{u}(t)$ at $t=t_{0}$ passes through the centre $\mathbf{z}$ and $\mathbf{u}\left(t_{0}\right) \neq \mathbf{z}$ (see Figure 10). Recall that the curvature can be computed from

$$
\kappa=\left[\mathbf{x}^{\prime}, \mathbf{x}^{\prime \prime}\right] /\left\|\mathbf{x}^{\prime}\right\|^{3},
$$

with the determinant $[\mathbf{p}, \mathbf{q}]=p_{1} q_{2}-p_{2} q_{1}$ of vectors $\mathbf{p}, \mathbf{q} \in \mathbb{R}^{2}$. A short computation indeed confirms that

$$
\left[\mathbf{x}^{\prime}, \mathbf{x}^{\prime \prime}\right]=2 w^{2}\left(u_{1}^{2}+\left(1+u_{2}\right)^{2}\right)\left[\mathbf{u}-\mathbf{z}, \mathbf{u}^{\prime}\right]
$$

Thus, at a regular curve point, zeros of the curvature are equivalent to linear dependency between $\mathbf{u}-\mathbf{z}$ and the tangent $\mathbf{u}^{\prime}$ of the preimage curve.

In the remainder of this paper we deal exclusively with $\mathrm{PH}$ curves of degree 7 , as these curves are capable of matching $G^{2}\left[C^{1}\right]$ Hermite boundary data. According to the representation of Lemma 2.1, there are four possibilities of constructing $\mathrm{PH}$ curves of degree 7: any choice of degrees $(m, k)=(i, 6-2 i)$ with $0 \leq i \leq 3$ is possible. However, the geometric flexibility of the $\mathrm{PH}$ curve depends mainly on the degree $m$ of the preimage curve $\mathbf{u}(t)$. For instance, the number of potential inflections equals the number of possible tangents that pass through the centre of 
control points of
the preimage curve

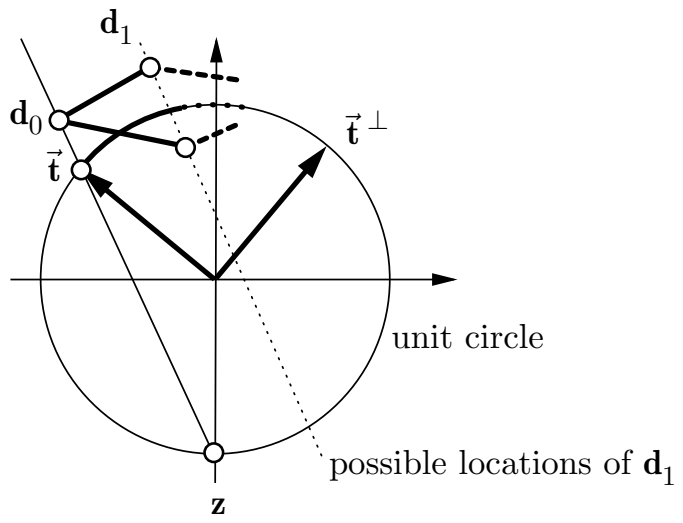

Figure 2. Lemma 2.5

projection. Thus, the choice $(m, k)=(3,0)$ seems to be the most appropriate one for our application, as the resulting $\mathrm{PH}$ curves of degree 7 can have the maximum number of inflections (i.e., 2), hence providing the maximum flexibility of shape. From now on we will consider $\mathrm{PH}$ curves with cubic preimage curve $\mathbf{u}(t)$ and a constant $w=1$.

The final lemma prepares the interpolation of $G^{2}\left[C^{1}\right]$ boundary data.

Lemma 2.5. Let the cubic preimage curve $\mathbf{u}(t)=\sum_{i=0}^{3} B_{i}^{3}(t) \mathbf{d}_{i}$ be given in Bézier form with the boundary control points

$$
\mathbf{d}_{0}=(1-\tau) \mathbf{z}+\tau \overrightarrow{\mathbf{t}}, \quad \mathbf{d}_{1}=\overrightarrow{\mathbf{t}}+\rho \overrightarrow{\mathbf{t}}^{\perp}+\nu(\overrightarrow{\mathbf{t}}-\mathbf{z})
$$

and with certain control points $\mathbf{d}_{2}, \mathbf{d}_{3} \in \mathbb{R}^{2}$. The unit vector $\overrightarrow{\mathbf{t}}=\{-\sin \phi, \cos \phi\}$ corresponds to a point on the unit circle, and $\overrightarrow{\mathbf{t}}^{\perp}=\{\cos \phi, \sin \phi\}$. As in the previous lemma, $\mathbf{z}=\{0,-1\}$ is the center of projection. The real parameters $\rho, \tau, \nu$ are arbitrary. Then, the boundary tangent and curvature of the resulting PH curve of degree 7 at $t=0$ are

$$
\mathbf{x}^{\prime}(0)=2 \tau^{2}(\cos \phi+1) \overrightarrow{\mathbf{t}} \quad \text { and } \quad \kappa(0)=-\frac{3 \rho}{2 \tau^{3}(\cos \phi+1)} .
$$

The proof results from straightforward calculations. An analogous observation can be derived for the opposite segment end point, i.e., at $t=1$.

According to this lemma, the first derivative and the the curvature can be interpolated by choosing the parameters $\tau$ and $\rho$. The remaining parameter $\nu$ is free: it influences neither $\mathbf{x}^{\prime}(0)$ nor $\kappa(0)$. That is, the second control point $\mathbf{d}_{1}$ of the cubic preimage curve can be moved on a parallel to $\overrightarrow{\mathbf{t}}-\mathbf{z}$ without altering the first derivative or curvature of the resulting $\mathrm{PH}$ cubic at $t=0$ (cf. Figure 2).

This observation will be used to facilitate the $G^{2}\left[C^{1}\right]$ Hermite interpolation problem with PH curves: curvatures and first derivatives at the segment boundaries will be interpolated by choosing the parameters $\tau, \rho$ (for $t=0$ ) and analogous parameters for $t=1$. This leaves the PH curve with two free parameters (one each for the inner control points $\mathbf{d}_{1}, \mathbf{d}_{2}$ of the preimage curve) which will then be used to match the given positional data. 


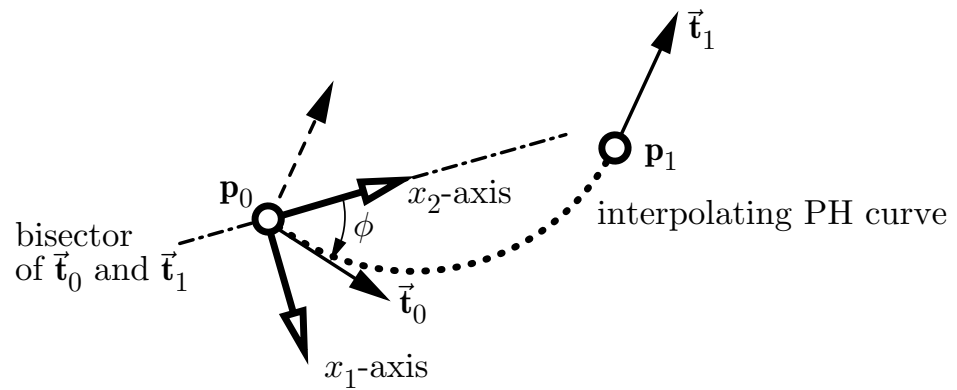

Figure 3. Adapted system of coordinates

\section{Hermite interpolation}

We construct a PH curve of degree 7, represented as a Bézier curve (1.1), that matches the following data:

- the boundary points $\mathbf{p}_{0}, \mathbf{p}_{1}$;

- the unit tangents $\overrightarrow{\mathbf{t}}_{0}, \overrightarrow{\mathbf{t}}_{1}$ and the lengths $l_{0}, l_{1}$ of the first derivatives at the boundary;

- the boundary curvatures $k_{0}, k_{1}$, cf. (2.5).

This leads to the interpolation conditions

$$
\begin{aligned}
& \mathbf{x}(0)=\mathbf{p}_{0}, \quad \mathbf{x}^{\prime}(0)=l_{0} \overrightarrow{\mathbf{t}}_{0}, \quad \kappa(0)=k_{0}, \\
& \mathbf{x}(1)=\mathbf{p}_{1}, \quad \mathbf{x}^{\prime}(1)=l_{1} \overrightarrow{\mathbf{t}}_{1}, \quad \text { and } \quad \kappa(1)=k_{1} .
\end{aligned}
$$

Throughout this section we are using an adapted system of coordinates, with the origin at $\mathbf{p}_{0}=\{0,0\}$ and with the $x_{2}$-axis spanned by the bisector of the unit tangents (see Figure 3). The direction of the $x_{1}$-axis is obtained by a rotation of $-\pi / 2$. Consequently, the given unit tangents may be represented as

$$
\overrightarrow{\mathbf{t}}_{0}=\{-s, c\} \quad \text { and } \quad \overrightarrow{\mathbf{t}}_{1}=\{s, c\},
$$

with $s=\sin \phi$ and $c=\cos \phi$, where $\phi$ is the oriented angle between the $x_{2}$-axis and the unit tangent $\overrightarrow{\mathbf{t}}_{0}$.

3.1. Finding the interpolants. The interpolating $\mathrm{PH}$ curve of degree 7 will be constructed with the help of the observations from Lemmas 2.1 and 2.5. The preimage curve of the stereographic projection is chosen as

$$
\mathbf{u}(t)=\left\{u_{1}(t), u_{2}(t)\right\}=\sum_{i=0}^{3} \mathbf{d}_{i} B_{i}^{3}(t)
$$

with the control points

$$
\begin{aligned}
& \mathbf{d}_{0}=\left(1-\tau_{0}\right) \mathbf{z}+\tau_{0} \overrightarrow{\mathbf{t}}_{0}, \quad \mathbf{d}_{1}=\overrightarrow{\mathbf{t}}_{0}+\rho_{0} \overrightarrow{\mathbf{t}}_{0}^{\perp}+\left(\mu_{0}+\mu_{1}\right)\left(\overrightarrow{\mathbf{t}}_{0}-\mathbf{z}\right), \\
& \mathbf{d}_{2}=\overrightarrow{\mathbf{t}}_{1}-\rho_{1} \overrightarrow{\mathbf{t}}_{1}^{\perp}+\left(\mu_{0}-\mu_{1}\right)\left(\overrightarrow{\mathbf{t}}_{1}-\mathbf{z}\right), \quad \text { and } \quad \mathbf{d}_{3}=\left(1-\tau_{1}\right) \mathbf{z}+\tau_{1} \overrightarrow{\mathbf{t}}_{1}
\end{aligned}
$$

that depend on the six unknown parameters $\tau_{i}, \rho_{i}$, and $\mu_{i}, i=0,1$.

The special representation (3.4) of the preimage control points is inspired by Lemma 2.5. In addition, we have split the two $\nu$-parameters (one for each segment boundary) into $\mu_{0} \pm \mu_{1}$. This simplifies the forthcoming calculations. It is possible 
to represent all cubic preimage curves in this way, provided that the resulting $\mathrm{PH}$ curves of degree 7 have the boundary unit tangents $\overrightarrow{\mathbf{t}}_{0}$ and $\overrightarrow{\mathbf{t}}_{1}$.

Once the parameters $\tau_{i}, \rho_{i}$, and $\mu_{i}$ have been found, the first derivative vector of the interpolating $\mathrm{PH}$ cubic results from (2.1),

$$
\mathbf{x}^{\prime}(t)=\left\{x_{1}^{\prime}(t), x_{2}^{\prime}(t)\right\}=\left\{2 u_{1}\left(1+u_{2}\right),\left(1+u_{2}\right)^{2}-u_{1}^{2}\right\}=\sum_{i=0}^{6} \mathbf{c}_{i} B_{i}^{6}(t) .
$$

Explicit formulas for the control points $\mathbf{c}_{i}=\left\{c_{i, 1}, c_{i, 2}\right\}$ can be generated easily with the help of computer algebra tools such as Maple or Mathematica. For the sake of brevity we omit these (somewhat lengthy) expressions.

Finally, the PH curve is given as a Bézier curve (1.1) of degree 7, with the control points $\mathbf{b}_{i}=\frac{1}{7} \sum_{j=0}^{i} \mathbf{c}_{j}$. In particular we obtain

$$
\mathbf{b}_{0}=\mathbf{x}(0)=\{0,0\} \quad \text { and } \quad \mathbf{b}_{7}=\mathbf{x}(1)=\left\{\vec{\mu}^{\top} Q \vec{\mu}, \vec{\mu}^{\top} R \vec{\mu}\right\}
$$

with the 3 -vector $\vec{\mu}=\left\{1, \mu_{0}, \mu_{1}\right\}^{\top}$. The two symmetric $3 \times 3$ matrices $Q=\left(q_{i, j}\right)$ and $R=\left(r_{i, j}\right)$ are listed in the appendix: they depend on the remaining parameters $\tau_{i}$ and $\rho_{i}$. The matrices satisfy

$$
q_{2,2}=q_{3,3}=0 \quad \text { and } \quad r_{2,3}=r_{3,2}=0 .
$$

These facts will make it very easy to compute the parameters $\mu_{0}, \mu_{1}$ by solving a single equation of degree 4. They are a consequence of the special choice (3.4).

According to Lemma 2.5 the parameters $\tau_{i}, \rho_{i}$ can be computed from the given curvature and derivative information at the boundaries, as follows.

- Firstly, the parameters $\tau_{i}$ are found from (2.8). This gives the two solutions

$$
\begin{array}{ll}
\tau_{0}^{(1)}=+\sqrt{\frac{1}{2} l_{0} /(c+1)}, & \tau_{1}^{(1)}=+\sqrt{\frac{1}{2} l_{1} /(c+1)}, \quad \text { and } \\
\tau_{0}^{(2)}=-\sqrt{\frac{1}{2} l_{0} /(c+1)}, & \tau_{1}^{(2)}=+\sqrt{\frac{1}{2} l_{1} /(c+1)} .
\end{array}
$$

The remaining two possible combinations of signs will be ignored, as they do not produce different solutions. This is due to the symmetry property of formula (2.1) (see Remark 2.4). The solutions arising from the first (resp. second) choice of signs will be called the solutions of the first (resp. second) kind. Note that for the solutions of the first (resp. second) kind, the preimage control points $\mathbf{d}_{0}$ and $\mathbf{d}_{3}$ are on the same side (resp. on different sides) of the centre $\mathbf{z}$. Thus, the solutions of the first kind can be expected to exhibit better shapes, as they should not get close to the centre $z$ of projection. The shape of solutions will be examined in more detail later.

- Secondly, the curvature parameters $\rho_{i}$ are computed from the given curvatures $k_{i}$

$$
\rho_{i}=-\frac{2}{3} k_{i} \tau_{i}^{3}(c+1) ; \quad i=0,1
$$

again, see (2.8).

The remaining two unknowns $\mu_{0}, \mu_{1}$ are found from the interpolation of the boundary points $\mathbf{p}_{0}=\{0,0\}$ (the origin of the coordinate system has been chosen there) and $\mathbf{p}_{1}=\left\{p_{1,1}, p_{2,2}\right\}$. The condition at the starting point is automatically satisfied 
(see (3.6)). The interpolation condition at the second point produces two quadratic equations

$$
\vec{\mu}^{\top} Q \vec{\mu}=p_{1,1} \quad \text { and } \quad \vec{\mu}^{\top} R \vec{\mu}=p_{1,2},
$$

with $\vec{\mu}=\left\{1, \mu_{0}, \mu_{1}\right\}^{\top}$. Owing to $q_{2,2}=q_{3,3}=0$, the first equation can immediately be solved for one of the unknowns, say $\mu_{0}$, giving a bilinear expression in $\mu_{1}$,

$$
\mu_{0}=-\frac{q_{1,1}+2 \mu_{1} q_{1,3}-p_{1,1}}{2 q_{1,2}+2 \mu_{1} q_{3,2}} .
$$

Substituting the result into the second equation leads to a single equation for the remaining variable. The numerator of this equation gives a quartic equation for $\mu_{1}$,

$$
\begin{aligned}
0 & =4 r_{3,3} q_{2,3}{ }^{2} \mu_{1}^{4}+\left(8 r_{1,3} q_{2,3}{ }^{2}+8 r_{3,3} q_{1,2} q_{2,3}\right) \mu_{1}^{3}+\left(4 r_{1,1} q_{2,3}{ }^{2}\right. \\
& \left.+4 q_{1,3}^{2} r_{2,2}-8 r_{1,2} q_{1,3} q_{2,3}-4 p_{1,2} q_{2,3}{ }^{2}+16 r_{1,3} q_{1,2} q_{2,3}+4 r_{3,3} q_{1,2}{ }^{2}\right) \mu_{1}^{2} \\
& +\left(8 r_{1,1} q_{1,2} q_{2,3}-8 r_{1,2} q_{1,3} q_{1,2}-8 p_{1,2} q_{1,2} q_{2,3}+8 r_{1,3} q_{1,2}{ }^{2}+4 q_{1,3} q_{1,1} r_{2,2}\right. \\
& \left.-4 p_{1,1} q_{1,3} r_{2,2}-4 r_{1,2} q_{1,1} q_{2,3}+4 r_{1,2} p_{1,1} q_{2,3}\right) \mu_{1}+4 r_{1,1} q_{1,2}{ }^{2}-2 r_{2,2} q_{1,1} p_{1,1} \\
& -4 r_{1,2} q_{1,1} q_{1,2}+r_{2,2} p_{1,1}{ }^{2}+4 r_{1,2} p_{1,1} q_{1,2}-4 p_{1,2} q_{1,2}{ }^{2}+r_{2,2} q_{1,1}{ }^{2} .
\end{aligned}
$$

This equation can now be solved either using appropriate numerical methods, or with the help of Ferrari's solution for the general quartic (see [3]). The latter approach is particularly useful for a theoretical analysis of the existence of solutions (see Section 4). For the practical implementation, however, it is more appropriate to rely on a numerical root-finding procedure that deals directly with the quadratic equations (3.10). The Taylor expansions (4.17) derived in Section 4 may be used in order to generate suitable initial values for the iterative numerical computations.

Equation (3.12) for $\mu_{1}$ may produce up to four real different solutions. After finding them, we substitute into (3.11) and compute the corresponding values of the first parameter $\mu_{0}$. In total, one may get up to eight different $\mathrm{PH}$ curves of degree 7 matching the given $G^{2}\left[C^{1}\right]$ boundary data, four for each kind of solutions (cf. (3.8)).

3.2. Number of solutions. In order to illustrate the Hermite interpolation problem we consider an example. Let the following tangent and curvature boundary data be given:

$$
\phi=\frac{\pi}{12}, \quad l_{0}=\frac{18}{10}, \quad l_{1}=1, \quad k_{0}=5, \quad k_{1}=7 .
$$

The angle $\phi$ specifies the directions of the boundary unit tangents $\overrightarrow{\mathbf{t}}_{0}, \overrightarrow{\mathbf{t}}_{1}$ with respect to the adapted system of coordinates (see (3.2)). From the given data we may find the values of the parameters $\tau_{0}, \tau_{1}$ and $\rho_{0}, \rho_{1}$ (see (3.8) and (3.9)). We obtain two quadruples of solutions, corresponding to the solutions of the first and second kind.

We will now discuss how many PH curves of degree 7 exist that interpolate these given boundary data, depending on the location of the segment end point $\mathbf{p}_{1}$. Note that $\mathbf{p}_{0}=\{0,0\}$, due to the adapted choice of coordinates.

We consider the feasible locations of the segment end point $\mathbf{x}(1)$, depending on the parameters $\mu_{0}, \mu_{1}$. They are obtained from the quadratic mapping $\mathbb{R}^{2} \rightarrow \mathbb{R}^{2}$

$$
\left(\mu_{0}, \mu_{1}\right) \mapsto \mathbf{x}(1)=\left\{\vec{\mu}^{\top} Q \vec{\mu}, \vec{\mu}^{\top} R \vec{\mu}\right\}
$$

(see (3.6)). In general, such a quadratic bivariate mapping maps the plane $\mathbb{R}^{2}$ to a subset of $\mathbb{R}^{2}$, which is multiply covered. A detailed study and classification of such mappings can be found, e.g., in [15]. 

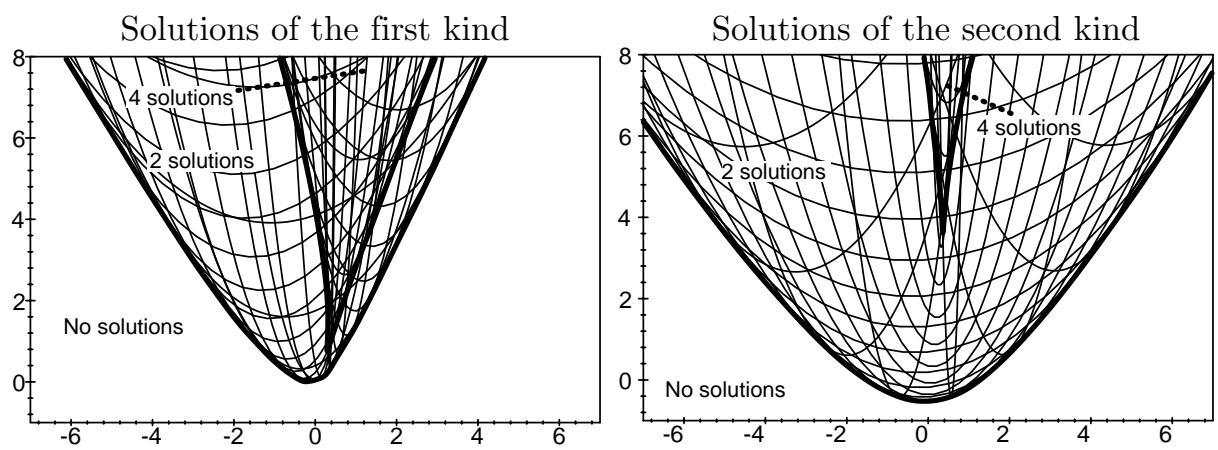

FiguRE 4. Number of solutions, depending on the location of $\mathbf{p}_{1}$.

In the above example, the situation is shown in Figure 4.

The left (resp. right) figure corresponds to the solutions of the first (resp. second) kind. The two families of parabolas (thin lines) are the images of the lines $\mu_{i}=$ constant in the $\mu_{0}, \mu_{1}$-plane. Their envelope is a quartic rational curve (thick lines); this curve separates the regions that lead to a different number of solutions.

The innermost region is covered four times by the quadratic mapping. Consequently, if the given segment end point $\mathbf{p}_{1}$ belongs to that region, the interpolation problem has four real solutions. The outer region is not a part of the range domain of the mapping (3.14). Thus, if $\mathbf{p}_{1}$ lies in this part of the plane, then there are no solutions of the interpolation problem available. In between both regions the image plane is covered twice, leading to two potential solutions.

3.3. Summary. The discussion of $G^{2}\left[C^{1}\right]$ Hermite interpolation leads to the following result.

Proposition 3.1. Consider the $G^{2}\left[C^{1}\right]$ Hermite interpolation problem with Pythagorean hodograph curves of degree 7 . Here, we restrict ourselves to PH curves which are obtained from a cubic preimage curve. Then, the interpolation problem may have up to eight real solutions. The interpolants can be computed with the help of the algorithm which is summarized in Table 1 .

Clearly, the proposition does not address the questions of existence and regularity of the solutions. This will be examined in the next section.

The Hermite interpolation procedure may produce several solutions; hence in applications it will be necessary to pick one of them. There are several possible criteria for this decision (examples follow).

- The distance (measured by a suitable norm) between the PH curves and a given curve. This measure is particularly useful if the Hermite data stem from an underlying given curve.

- The "fairness" or the "bending energy" of the PH curves. This is measured by expressions such as $\int_{s_{0}}^{s_{1}} \kappa^{2} d s$ or, as an approximation to the latter, $\int_{0}^{1}\left\|\mathbf{x}^{\prime \prime}(t)\right\|^{2} d t$.

- The arc length of the PH curves.

Clearly, the choice of the appropriate functional depends on the specific application. 
TABle 1. The $G^{2}\left[C^{1}\right]$ Hermite interpolation algorithm.

1) Transform the $G^{2}\left[C^{1}\right]$ Hermite boundary data into the adapted system of coordinates.

2) Compute the parameters $\tau_{i}^{(j)}$ and $\rho_{i}$ from the given boundary curvatures $k_{i}$ and the lengths of the first derivatives $l_{i}, i=0,1, j=1,2$ (see (3.8) and (3.9)).

3) Find the parameters $\mu_{0}, \mu_{1}$ by solving the two quadratic equations (3.10). This may lead to up to eight different solutions, four each for the solutions of the first (resp. second) kind.

4) Compute the control points $\mathbf{b}_{i}$ of the interpolating PH curves (see equations (3.3)-(3.6) ).

5) Transform the interpolating PH curves back into world coordinates.

a) Hermite interpolants
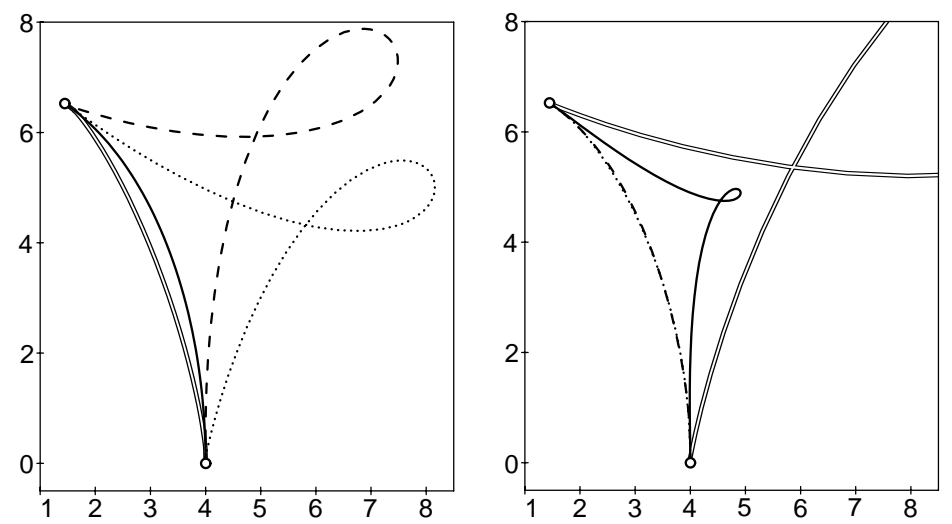

b) Curvature plots
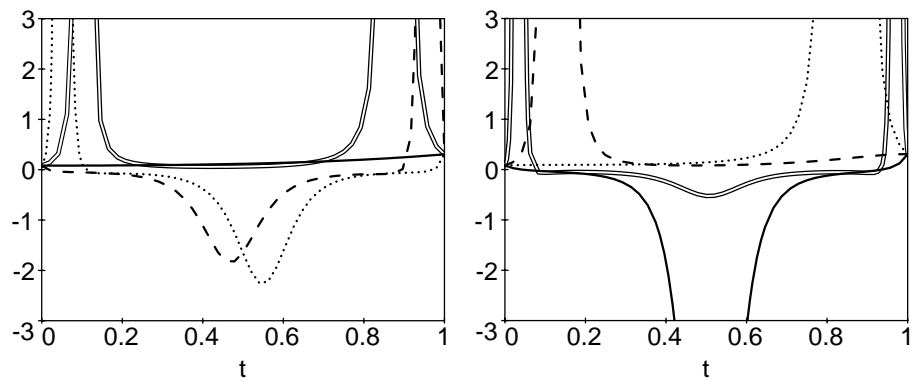

Figure 5. (a) The eight solutions of the $G^{2}\left[C^{1}\right]$-Hermite interpolation problem, and (b) the associated curvature plots. 
Example 3.2. We consider $G^{2}\left[C^{1}\right]$ data which have been taken from an arc of an ellipse. Applying the Hermite interpolation procedure to these data leads to eight distinct PH curves (see Figure $5 \mathrm{a}$ ). The solutions of the first and the second kind are shown in the right and left figures, respectively. There are four solutions each, visualized by different line styles ("hollow", "solid", "dashed", and "dotted"). Note that the dotted and the dashed curves in the right figure are almost identical.

In order to judge the quality of the solutions, the associated curvature plots are provided in the second line of the figure. As shown by these plots, only one of these solutions behaves as expected (the solid black curve in the left figure). This $\mathrm{PH}$ curve is virtually indistinguishable from the original elliptic arc (not shown). The remaining seven curves exhibit singular points and/or almost singular points, as indicated by the poles of the curvature function.

The next section studies the behaviour of the solutions in more detail.

\section{Asymptotic SOLVABILITy AND ASYMPtotic BEHAVIOUR}

The $G^{2}\left[C^{1}\right]$ Hermite interpolation problem from the previous section does not necessarily have regular real solutions. Based on Ferrari's solution of the general quartic, one could formulate criteria that guarantee the existence of real solutions. These criteria, however, would give very little geometrical insight, as the resulting expressions are rather technical and complicated. Instead we consider the asymptotic behaviour of the solutions. More precisely, assuming that the $G^{2}\left[C^{1}\right]$ Hermite data are taken from an arc of an analytical curve, we investigate the asymptotic behaviour of solvability and shape of solutions for decreasing stepsize. In order to get results which can be interpreted geometrically, we use the so-called canonical expansion of the curve from differential geometry, as follows.

4.1. Canonical Taylor expansion. Consider a planar parametric curve. It is assumed to be analytical in a neighborhood of one of its points. Let $\overline{\mathbf{p}}(S)=$ $\left\{\bar{p}_{1}(S), \bar{p}_{2}(S)\right\}$ be the parametric representation of the curve with respect to the arc length $S$; it is characterized by $\left\|\overline{\mathbf{p}}^{\prime}(S)\right\| \equiv 1$. Differentiation gives the unit tangent

$$
\overline{\mathbf{p}}^{\prime}(S)=\overrightarrow{\mathbf{t}}(S) \text {. }
$$

Its derivatives are governed by the Frenet-Serret formulas

$$
\overrightarrow{\mathbf{t}}^{\prime}(S)=\kappa(S) \overrightarrow{\mathbf{n}}(S) \quad \text { and } \quad \overrightarrow{\mathbf{n}}^{\prime}(S)=-\kappa(S) \overrightarrow{\mathbf{t}}(S),
$$

where $\overrightarrow{\mathbf{n}}(S)$ and $\kappa(S)$ are the unit normal and the curvature at $\overline{\mathbf{p}}(S)$. At each point $\overline{\mathbf{p}}(S)$, unit tangent and unit normal form a positively oriented base of $\mathbb{R}^{2}$. Consequently, points on curves with positive curvature travel counterclockwise for increasing arc-length parameter $S$.

Starting from the Taylor expansion of $\overline{\mathbf{p}}(S)$,

$$
\overline{\mathbf{p}}(S)=\overline{\mathbf{p}}(0)+\overline{\mathbf{p}}^{\prime}(0) S+\frac{1}{2} \overline{\mathbf{p}}^{\prime \prime}(0) S^{2}+\frac{1}{6} \overline{\mathbf{p}}^{\prime \prime \prime}(0) S^{3}+\mathcal{O}\left(S^{4}\right),
$$

and with the help of the Frenet-Serret formulas, one may successively replace the derivatives of $\overline{\mathbf{p}}(S)$ at $S=0$ with certain linear combinations of $\overrightarrow{\mathbf{t}}(0)$ and $\overrightarrow{\mathbf{n}}(0)$. Expressions for the higher order derivatives of $\overline{\mathbf{p}}$ are obtained by differentiating the Frenet-Serret formulas. Clearly, this generates expressions involving the derivatives of the curvature $\kappa(S)$. 
Combining these formulas, one gets the so-called canonical Taylor expansion with respect to the arc length parameterization $\overline{\mathbf{p}}(S)=\left\{\bar{p}_{1}(S), \bar{p}_{2}(S)\right\}$ with

$$
\begin{aligned}
& \bar{p}_{1}(S)=S-\frac{1}{6} \kappa_{0}^{2} S^{3}-\frac{1}{8} \kappa_{0} \kappa_{1} S^{4}+\mathcal{O}\left(S^{5}\right) \text { and } \\
& \bar{p}_{2}(S)=\frac{1}{2} \kappa_{0} S^{2}+\frac{1}{6} \kappa_{1} S^{3}+\frac{1}{24}\left(\kappa_{2}-\kappa_{0}^{3}\right) S^{4}+\mathcal{O}\left(S^{5}\right) .
\end{aligned}
$$

Here, the system of coordinates has been chosen such that $\overrightarrow{\mathbf{t}}(0)=\{1,0\}^{\top}$ and $\overrightarrow{\mathbf{n}}(0)=\{0,1\}^{\top}$, with the origin at $\overline{\mathbf{p}}(0)$. The coefficient $\kappa_{i}$ is the $i$ th derivative of the curvature with respect to the arc length parameter at $S=0$.

For more information the reader should consult any textbook on elementary differential geometry, e.g., [11, $\S \S 9,15$ and 18].

In computer-aided design applications, however, curves are generally not parameterized by the arc length (cf. [7). In order to obtain a parametric representation of the given curve with respect to a general parameter $T$, we apply the substitution $S=S(T)$ with $S(0)=0$. Again, in a neighbourhood of $T=0$, the substitution is represented by its Taylor expansion with respect to the new curve parameter $T$,

$$
S(T)=r_{1} T+\frac{1}{2} r_{2} T^{2}+\frac{1}{6} r_{3} T^{3}+\frac{1}{24} r_{4} T^{4}+\mathcal{O}\left(T^{5}\right)
$$

with certain coefficients $r_{i} \in \mathbb{R}$. Without loss of generality we may assume that $r_{1}=1$ holds; hence $\left\|\mathbf{p}^{\prime}(0)\right\|=1$. This normalization can be achieved by a suitable scaling of the original curve.

By combining (4.4) and (4.5) we obtain the Taylor expansion of the given curve

$$
\mathbf{p}(T)=\left\{p_{1}(T), p_{2}(T)\right\}=\left\{\bar{p}_{1}(S(T)), \bar{p}_{2}(S(T))\right\}
$$

at $T=0$, as summarized in

Proposition 4.1. Any given curve $\mathbf{p}=\mathbf{p}(T), T \in[a, b] \subseteq \mathbb{R}$, which is assumed to be analytical in a neighbourhood of $T=0$, can (after switching to suitable Cartesian coordinates and possible scaling) locally be approximated by the Taylor expansion

$$
\begin{aligned}
p_{1}(T)= & T+\frac{1}{2} r_{2} T^{2}+\left(\frac{1}{6} r_{3}-\frac{1}{6} \kappa_{0}^{2}\right) T^{3} \\
& +\left(\frac{1}{24} r_{4}-\frac{1}{4} \kappa_{0}^{2} r_{2}-\frac{1}{8} \kappa_{0} \kappa_{1}\right) T^{4}+\mathcal{O}\left(T^{5}\right), \\
p_{2}(T)= & \frac{1}{2} \kappa_{0} T^{2}+\left(\frac{1}{2} \kappa_{0} r_{2}+\frac{1}{6} \kappa_{1}\right) T^{3} \\
& +\left(\frac{1}{6} \kappa_{0} r_{3}+\frac{1}{8} \kappa_{0} r_{2}{ }^{2}+\frac{1}{4} \kappa_{1} r_{2}+\frac{1}{24} \kappa_{2}-\frac{1}{24} \kappa_{0}^{3}\right) T^{4}+\mathcal{O}\left(T^{5}\right) .
\end{aligned}
$$

The coefficients $\kappa_{i}$ are the derivatives of the curvature with respect to the arc length parameter at $S=0$. The coefficients $r_{i}$ are the derivatives of the arc length function $S=S(T)=\int_{0}^{T}\left\|\mathbf{p}^{\prime}(\tau)\right\| d \tau$ at $T=0$, where $r_{1}=1$ is assumed.

This Taylor expansion serves as the basic tool for studying the asymptotic behaviour of solvability and of the solutions of the Hermite interpolation problem. By combining the canonical Taylor expansion (4.4), specifying the geometry of the given curve, with the general parameterization (4.5), we are able to study both the influence of the geometry and of the parameterization. However, in order to make the result of the Hermite interpolation procedure independent of the initial parameterization of the given curve, one may wish to choose its arc-length parameterization. In the results derived below, this is achieved by choosing $r_{2}=r_{3}=\cdots=0$. On the other hand, in many applications it may be desirable to approximate both the given curve and its parameterization with a $\mathrm{PH}$ curve. This corresponds to using the more general representation (4.7). 
4.2. Asymptotical Hermite interpolation. Consider the segment $T \in[0, \Delta]$ of an analytical curve $\mathbf{p}(T)$ for some stepsize $\Delta>0$. Using the local parameter $t \in[0,1]$, this segment is parameterized as $\mathbf{p}(t \Delta)$. It produces the following $G^{2}\left[C^{1}\right]$ Hermite boundary data.

$$
\begin{array}{ll}
\mathbf{p}_{0}=\mathbf{p}(0), & \mathbf{p}_{1}=\mathbf{p}(\Delta), \\
\overrightarrow{\mathbf{t}}_{0}=\mathbf{p}^{\prime}(0) /\left\|\mathbf{p}^{\prime}(0)\right\|, & \overrightarrow{\mathbf{t}}_{1}=\mathbf{p}^{\prime}(\Delta) /\left\|\mathbf{p}^{\prime}(\Delta)\right\|, \\
l_{0}=\Delta\left\|\mathbf{p}^{\prime}(0)\right\|, & l_{1}=\Delta\left\|\mathbf{p}^{\prime}(\Delta)\right\|, \\
k_{0}=\left[\mathbf{p}^{\prime}(0), \mathbf{p}^{\prime \prime}(0)\right] /\left\|\mathbf{p}^{\prime}(0)\right\|^{3}, & k_{1}=\left[\mathbf{p}^{\prime}(\Delta), \mathbf{p}^{\prime \prime}(\Delta)\right] /\left\|\mathbf{p}^{\prime}(\Delta)\right\|^{3} .
\end{array}
$$

We apply the Hermite interpolation procedure with $\mathrm{PH}$ curves of degree 7 to these data (see Proposition 3.1 and Table 1).

Question 4.2. How do the solvability of the interpolation problem and the shape of the solutions behave for decreasing stepsize $\Delta \rightarrow 0+$ ?

In order to answer this question, we generate Taylor expansion for all quantities that are involved in the previously described Hermite interpolation procedure. This is most efficiently done with the help of suitable computer algebra tools. Our results rely on the Maple V system. We sketch some steps of the computations. In order to be as brief as possible, we give only a few shortened Taylor expansions for some of the required variables.

As the very first step, one has to generate Taylor expansions for the input data (4.8). In order to apply the Hermite interpolation procedure from the previous section, these data have to be transformed into the adapted system of coordinates. After this transformation, the segment end points are $\mathbf{p}_{0}=\{0,0\}$ and $\mathbf{p}_{1}=\left\{p_{1,1}, p_{1,2}\right\}$ with

$$
\begin{aligned}
& p_{1,1}=\frac{1}{12} \kappa_{1} \Delta^{3}+\left(\frac{1}{24} \kappa_{2}+\frac{1}{8} \kappa_{1} r_{2}\right) \Delta^{4}+\mathcal{O}\left(\Delta^{5}\right) \\
& p_{1,2}=\Delta+ \frac{1}{2} r_{2} \Delta^{2}+\left(\frac{1}{6} r_{3}-\frac{1}{24} \kappa_{0}^{2}\right) \Delta^{3} \\
& \quad\left(\frac{1}{24} r_{4}-\frac{1}{16} \kappa_{0}^{2} r_{2}-\frac{1}{24} \kappa_{0} \kappa_{1}\right) \Delta^{4}+\mathcal{O}\left(\Delta^{5}\right)
\end{aligned}
$$

The directions of the boundary unit tangents $\overrightarrow{\mathbf{t}}_{0}, \overrightarrow{\mathbf{t}}_{1}$ are specified by the sine and cosine functions

$$
\begin{aligned}
& s=-\frac{1}{2} \kappa_{0} \Delta-\left(\frac{1}{4} \kappa_{0} r_{2}+\frac{1}{4} \kappa_{1}\right) \Delta^{2} \\
& +\left(\frac{1}{48} \kappa_{0}^{3}-\frac{1}{12} \kappa_{0} r_{3}-\frac{1}{4} \kappa_{1} r_{2}-\frac{1}{12} \kappa_{2}\right) \Delta^{3}+\mathcal{O}\left(\Delta^{4}\right), \\
& c=1-\frac{1}{8} \kappa_{0}^{2} \Delta^{2}+\left(-\frac{1}{8} \kappa_{0}^{2} r_{2}-\frac{1}{8} \kappa_{0} \kappa_{1}\right) \Delta^{3}+\mathcal{O}\left(\Delta^{4}\right)
\end{aligned}
$$

(see (3.2)). The lengths of the boundary tangents and the boundary curvatures have the expansions

$$
\begin{array}{ll}
l_{0}=\Delta, & l_{1}=\Delta+r_{2} \Delta^{2}+\frac{r_{3}}{2} \Delta^{3}+\frac{r_{4}}{6} \Delta^{4}+\mathcal{O}\left(\Delta^{5}\right), \\
k_{0}=\kappa_{0}, & \text { and } \quad k_{1}=\kappa_{0}+\kappa_{1} \Delta+\left(\frac{1}{2} \kappa_{1} r_{2}+\frac{1}{2} \kappa_{2}\right) \Delta^{2}+\mathcal{O}\left(\Delta^{3}\right) .
\end{array}
$$

During the discussion of the asymptotic behaviour we have to examine the case of a curved point $\left(\kappa_{0} \neq 0\right)$ and an inflection or flat point $\left(\kappa_{0}=0\right)$ separately.

4.3. The case $\kappa_{0} \neq 0$. At first we consider the solutions of the first kind.

Proposition 4.3. Assume that the original curve has nonvanishing curvature at the point $\mathbf{p}(0)$, i.e., $\kappa_{0} \neq 0$. If the technical assumption

$$
\kappa_{0} r_{2}+2 \kappa_{1} \neq 0
$$


is satisfied, and provided that the stepsize $\Delta$ is sufficiently small, then the $G^{2}\left[C^{1}\right]$ Hermite interpolation problem with $\mathrm{PH}$ curves of degree 7 has exactly four real solutions of the first kind. These solutions have Taylor expansions

$$
\mathbf{x}^{(j)}(t)=\left\{x_{1}^{(j)}(t) \kappa_{0} \Delta^{2}+\mathcal{O}\left(\Delta^{3}\right), x_{2}^{(j)}(t) \Delta+\mathcal{O}\left(\Delta^{2}\right)\right\}, \quad j=1, \ldots, 4,
$$

with certain polynomials $x_{1}^{(j)}, x_{2}^{(j)}$ of degree 7 not depending on the coefficients $\kappa_{i}$ or $r_{i}$. In particular, $x_{1}^{(3)}=\frac{1}{2} t-\frac{1}{2} t^{2}$ and $x_{2}^{(3)}=t$.

Remark 4.4. Due to space limitations we do not present explicit formulas for all solutions $\mathbf{x}^{(j)}(t)$. Instead, they are visualized in Figure 6] (see the explanations below). As observed later, the solution for $j=3$ seems to be the most useful one.

Proof. From (3.8) and (3.9) we obtain the following Taylor expansions with respect to powers of $\sqrt{\Delta}$ :

$$
\begin{aligned}
\tau_{0}= & \frac{1}{2} \sqrt{\Delta}+\frac{1}{64} \kappa_{0}^{2} \Delta^{5 / 2}+\left(\frac{1}{64} \kappa_{0}^{2} r_{2}+\frac{1}{64} \kappa_{0} \kappa_{1}\right) \Delta^{7 / 2}+\mathcal{O}\left(\Delta^{9 / 2}\right), \\
\tau_{1}= & \frac{1}{2} \sqrt{\Delta}+\frac{1}{4} r_{2} \Delta^{3 / 2}+\left(\frac{1}{8} r_{3}+\frac{1}{64} \kappa_{0}^{2}-\frac{1}{16} r_{2}^{2}\right) \Delta^{5 / 2}, \\
& +\left(\frac{1}{24} r_{4}+\frac{3}{128} \kappa_{0}^{2} r_{2}+\frac{1}{64} \kappa_{0} \kappa_{1}+\frac{1}{32} r_{2}^{3}-\frac{1}{16} r_{2} r_{3}\right) \Delta^{7 / 2}+\mathcal{O}\left(\Delta^{9 / 2}\right), \\
\rho_{0}= & -\frac{1}{6} \kappa_{0} \Delta^{3 / 2}-\frac{1}{192} \kappa_{0}^{3} \Delta^{7 / 2}+\mathcal{O}\left(\Delta^{9 / 2}\right), \\
\rho_{1}= & -\frac{1}{6} \kappa_{0} \Delta^{3 / 2}+\left(-\frac{1}{4} \kappa_{0} r_{2}-\frac{1}{6} \kappa_{1}\right) \Delta^{5 / 2} \\
& +\left(-\frac{1}{192} \kappa_{0}^{3}-\frac{1}{8} \kappa_{0} r_{3}-\frac{1}{16} \kappa_{0} r_{2}^{2}-\frac{1}{3} \kappa_{1} r_{2}-\frac{1}{12} \kappa_{2}\right) \Delta^{7 / 2}+\mathcal{O}\left(\Delta^{9 / 2}\right) .
\end{aligned}
$$

Equation (3.12) gives a quartic equation for the unknown parameter $\mu_{1}$ :

$$
\frac{3456}{42875} \kappa_{0}{ }^{2} \mu_{1}{ }^{4}+\left(\frac{3456}{42875} \kappa_{0}{ }^{2} \mu_{1}{ }^{4} r_{2}+\frac{3456}{42875} \kappa_{0} \mu_{1}{ }^{4} \kappa_{1}-\frac{342}{875} \mu_{1}{ }^{2} \kappa_{0}{ }^{2}\right) \Delta^{4}+\mathcal{O}\left(\Delta^{5}\right) .
$$

(Only the very first terms of the expansion are shown.) We solve this equation with the help of Ferrari's solution of the general quartic (see [3]). First we consider the associated cubic resolvent and solve it via Cardan's solution of the general cubic. This involves generating a certain discriminant. If the discriminant is negative, then the cubic resolvent has three real roots. The Taylor expansion of the discriminant is

$$
D=-\frac{2373434722791475}{1014454095446016} \frac{\left(\kappa_{0} r_{2}+2 \kappa_{1}\right)^{2}}{\kappa_{0}^{2}} \Delta^{8}+\mathcal{O}\left(\Delta^{9}\right),
$$

hence real solutions are guaranteed to exist, provided that the conditions $\kappa_{0} \neq 0$ and (4.12) are satisfied. Similarly, by generating their Taylor expansions, the roots of the cubic resolvent can be shown to be nonnegative, provided that the stepsize $\Delta$ is sufficiently small. Then, as a consequence from Ferrari's formulas, we obtain four real solutions for $\mu_{1}^{(j)}$. Similarly it can be shown that four associated solutions $\mu_{0}^{(j)}$ exist.

We generate Taylor expansions of the four solution pairs $\left(\mu_{0}^{(j)}, \mu_{1}^{(j)}\right)$, as follows. The leading terms of the Taylor expansions are obtained with the help of Cardan's and Ferrari's formulas. Then the higher order terms can be generated by substituting them in the expansions of the interpolation conditions (3.10). 

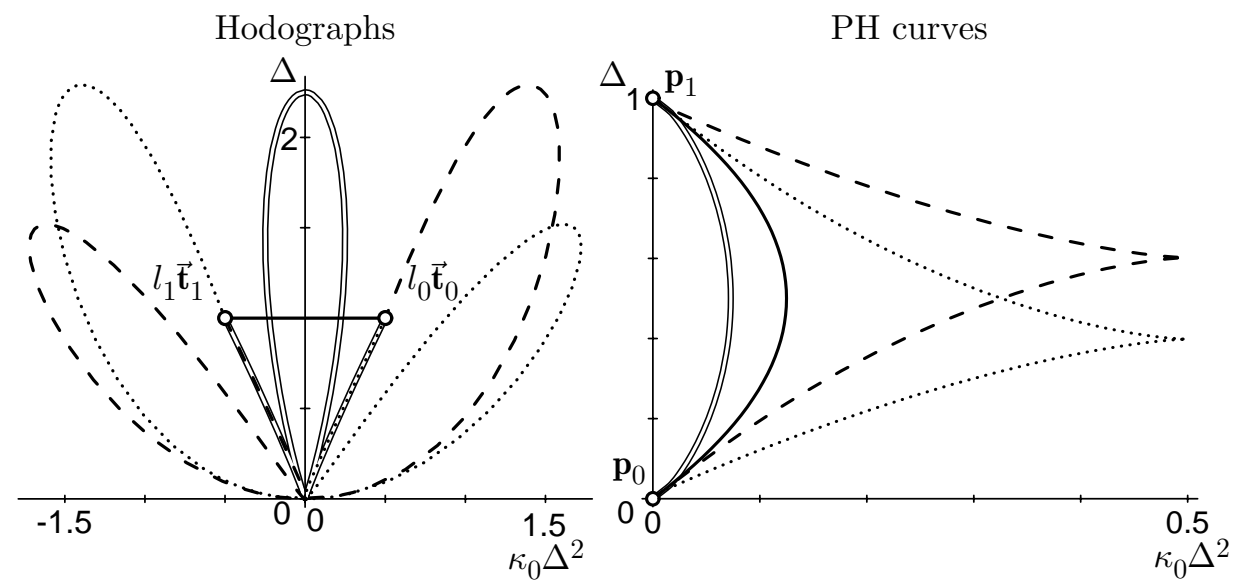

Figure 6. Asymptotic behaviour of the solutions of the first kind (right) $\mathbf{x}^{(j)}(t)$ and of their hodographs $\mathbf{x}^{(j) \prime}(t)$ (left). The solutions 1-4 correspond to the line styles "hollow", "dotted", "solid", and "dashed", respectively.

Summing up, we obtain Taylor expansions for the four pairs of solutions $\left(\mu_{0}^{(j)}, \mu_{1}^{(j)}\right)$ :

$$
\begin{array}{ll}
\mu_{0}^{(1)}=-1-\frac{7}{6} \sqrt{\Delta}+\mathcal{O}\left(\Delta^{3 / 2}\right), & \mu_{1}^{(1)}=-\frac{140 \kappa_{1}+89 \kappa_{0} r_{2}}{456 \kappa_{0}} \Delta^{3 / 2}+\mathcal{O}\left(\Delta^{5 / 2}\right), \\
\mu_{0}^{(2)}=-1-\frac{3}{8} \sqrt{\Delta}+\mathcal{O}\left(\Delta^{3 / 2}\right), & \mu_{1}^{(2)}=-\frac{7 \sqrt{57}}{24} \sqrt{\Delta}+\mathcal{O}\left(\Delta^{3 / 2}\right), \\
\mu_{0}^{(3)}=-1+\frac{1}{2} \sqrt{\Delta}+\mathcal{O}\left(\Delta^{3 / 2}\right), & \mu_{1}^{(3)}=-\frac{1}{24} r_{2} \Delta^{3 / 2}+\mathcal{O}\left(\Delta^{5 / 2}\right), \\
\mu_{0}^{(4)}=-1-\frac{3}{8} \sqrt{\Delta}+\mathcal{O}\left(\Delta^{3 / 2}\right), & \mu_{1}^{(4)}=\frac{7 \sqrt{57}}{24} \sqrt{\Delta}+\mathcal{O}\left(\Delta^{3 / 2}\right) .
\end{array}
$$

Now we substitute the expansions (4.10), (4.14), (4.17) into the equations (3.2)(3.5)). This produces Taylor expansions of the hodographs $\mathbf{x}^{\prime}(t)$ of the four interpolating $\mathrm{PH}$ curves, of the form

$$
\mathbf{x}^{(j) \prime}(t)=\left\{x_{1}^{(j) \prime}(t) \kappa_{0} \Delta^{2}+\mathcal{O}\left(\Delta^{3}\right), x_{2}^{(j) \prime}(t) \Delta+\mathcal{O}\left(\Delta^{2}\right)\right\}, \quad j=1, \ldots, 4,
$$

with certain polynomials $x_{1}^{(j) \prime}, x_{2}^{(j) \prime}$ of degree 6 not depending on the coefficients $\kappa_{i}$ or $r_{i}$. Finally, integrating these hodographs with respect to $t$ gives the Taylor expansions (4.13) of the interpolating $\mathrm{PH}$ curves themselves.

The asymptotic shapes of the hodographs and of the $\mathrm{PH}$ curves are shown in Figure 6. We have plotted the curves $\left\{x_{1}^{(j) \prime}(t), x_{2}^{(j) \prime}(t)\right\}$ and $\left\{x_{1}^{(j)}(t), x_{2}^{(j)}(t)\right\}$, $t \in[0,1]$, that are generated by the leading terms of the Taylor expansions. If the stepsize $\Delta$ becomes sufficiently small, then the shapes of the four solutions and of their hodographs get more and more similar to the curves which are shown in the figure.

At the boundaries $t \in\{0,1\}$, the hodographs match the leading terms of the prescribed boundary tangents

$$
\begin{aligned}
& l_{0} \overrightarrow{\mathbf{t}}_{0}=\left\{\frac{1}{2} \kappa_{0} \Delta^{2}+\mathcal{O}\left(\Delta^{3}\right), \Delta+\mathcal{O}\left(\Delta^{2}\right)\right\}, \\
& l_{1} \overrightarrow{\mathbf{t}}_{1}=\left\{-\frac{1}{2} \kappa_{0} \Delta^{2}+\mathcal{O}\left(\Delta^{3}\right), \Delta+\mathcal{O}\left(\Delta^{2}\right)\right\}
\end{aligned}
$$



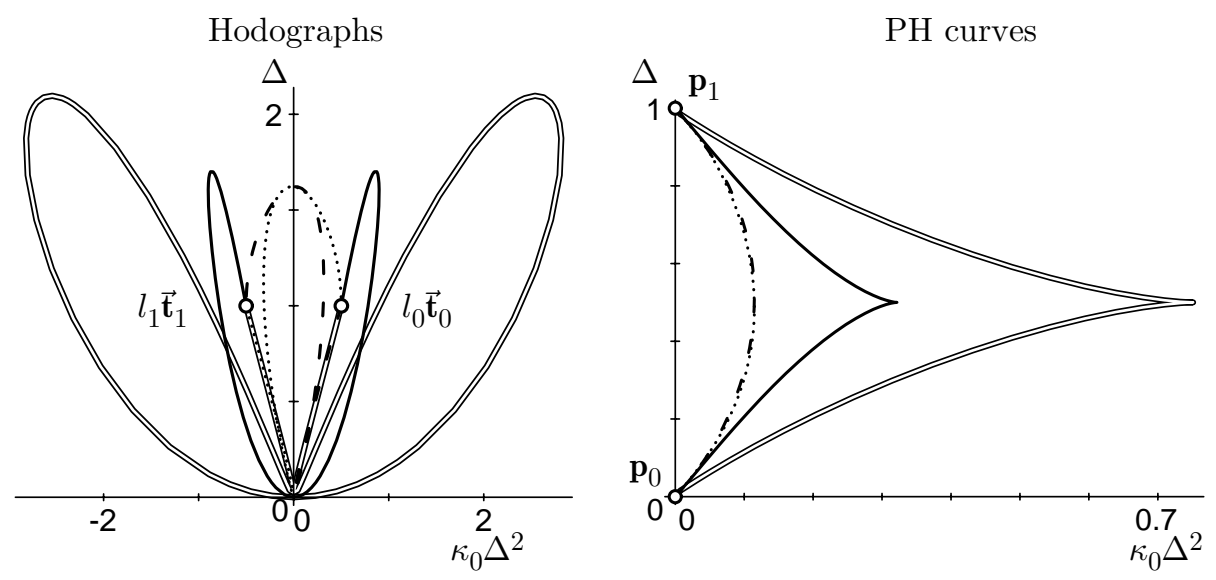

Figure 7. Asymptotic behaviour of the solutions of the second kind (right) $\mathbf{x}(t)$ and of their hodographs $\mathbf{x}^{\prime}(t)$ (left).

(cf. (3.2), (4.10), and (4.11)). Similarly, the PH curves interpolate the leading terms of the boundary points $\mathbf{p}_{i}$ (see (4.9)). The solutions 1,2 and 4 lead asymptotically to $\mathrm{PH}$ curves of degree 7 with two singularities (cusps) each, corresponding to the simultaneous roots of the components of the hodograph. Some of these singularities are hard to detect in the right figure, but their existence can clearly be seen from the hodographs. The third solution, however, gives a regular curve - it leads to the best approximation of the original curve among the four solutions.

Note the similarity between the asymptotic shapes of the interpolants (Figure 6 right) and the curves which are obtained in the example (Figure 5a, left)! Decreasing the stepsize $\Delta$ would pull the loops of the examples into the cusps of the asymptotic shapes.

The solutions of the second kind can be discussed in an analogous way.

Proposition 4.5. Assume again that the original curve has nonvanishing curvature at $\mathbf{p}(0)$, i.e., $\kappa_{0} \neq 0$. If the technical assumption

$$
13 \kappa_{0} r_{2}+34 \kappa_{1} \neq 0
$$

is satisfied, and provided that the stepsize $\Delta$ is sufficiently small, then the $G^{2}\left[C^{1}\right]$ Hermite interpolation problem with $\mathrm{PH}$ curves of degree 7 has exactly four real solutions of the second kind. These solutions have Taylor expansions

$$
\mathbf{x}^{(j)}(t)=\left\{x_{1}^{(j)}(t) \kappa_{0} \Delta^{2}+\mathcal{O}\left(\Delta^{3}\right), x_{2}^{(j)}(t) \Delta+\mathcal{O}\left(\Delta^{2}\right)\right\}, \quad j=5, \ldots, 8,
$$

with certain polynomials $x_{1}^{(j)}, x_{2}^{(j)}$ of degree 7 not depending on the coefficients $\kappa_{i}$ or $r_{i}$.

The asymptotic shapes of the four hodographs and the corresponding $\mathrm{PH}$ curves are shown in Figure 7 Again, we have plotted the curves which are generated by the leading terms of the Taylor expansions. The hollow interpolant has got three singularities, the remaining three solutions have only one singularity each. Note again the similarity between the asymptotic shapes of the interpolants (Figure 7 right) and the curves which are obtained in the example (Figure $5 \mathrm{~b}$, right). 
Remark 4.6. We conjecture that the technical assumptions (4.12), (4.20) can be eliminated from the discussion of solutions. By taking into acocunt the higher order terms of the Taylor expansions of the cubic discriminants $D$, it should be possible to conclude the existence of four real solutions for each kind of solution, even if the conditions

$$
\kappa_{0} r_{2}+2 \kappa_{1} \neq 0 \quad \text { or } 13 \kappa_{0} r_{2}+34 \kappa_{1} \neq 0
$$

are violated.

Note that the conditions (4.22) have a geometric meaning also. If the original curve is given by its arc-length parameterization $S(t) \equiv t$ (cf. 4.5 ), then they are violated at points with stationary curvature $\left(\kappa_{1}=0\right)$, the so-called vertices or cyclic points. For instance, if the Hermite data are taken from a circle in arc-length parameterization, then both conditions are violated. Moreover, in this case it can be shown that the cubic discriminant is even identically zero! In this case, the cubic resolvents have real solutions with multiplicity greater than 1 . Nevertheless, if the stepsize gets sufficiently small, then the interpolation problem leads to eight real solutions, with the same asymptotic shapes as discussed earlier.

The remaining condition $\kappa_{0} \neq 0$ (which is violated at inflections of the original curve) cannot be eliminated; it requires a separate analysis.

4.4. The case $\kappa_{0}=0$. So far, the case $\kappa_{0}=0$ (which occurs at inflection or flat points of the original curve) had to be excluded. In this case it is possible to give a similar analysis of the existence and behaviour of solutions. In the case of the solutions of the first kind we obtain:

Proposition 4.7. Assume that the original curve has a nonflat inflection point at $\mathbf{p}(0)$, i.e., $\kappa_{0}=0$ and $\kappa_{1} \neq 0$. If the stepsize $\Delta$ is sufficiently small, then the $G^{2}\left[C^{1}\right]$ Hermite interpolation problem with $P H$ curves of degree 7 has exactly four real solutions of the first kind. These solutions have Taylor expansions

$$
\hat{\mathbf{x}}^{(j)}(t)=\left\{\hat{x}_{1}^{(j)}(t) \kappa_{1} \Delta^{3}+\mathcal{O}\left(\Delta^{4}\right), \hat{x}_{2}^{(j)}(t) \Delta+\mathcal{O}\left(\Delta^{2}\right)\right\}, \quad j=1, \ldots, 4,
$$

with certain polynomials $\hat{x}_{1}^{(j)}, \hat{x}_{2}^{(j)}$ of degree 7 not depending on the coefficients $\kappa_{i}$ or $r_{i}$. In particular, $\hat{x}_{1}^{(3)}=\frac{1}{4} t-\frac{1}{8} t^{3}$ and $\hat{x}_{2}^{(3)}=t$.

Again, only the third solution has the desired shape, without singularities. The results are illustrated in Figure 8 At the boundaries $t \in\{0,1\}$, each of the four hodographs matches the leading terms of the prescribed boundary tangents

$$
\begin{aligned}
& l_{0} \overrightarrow{\mathbf{t}}_{0}=\left\{\frac{1}{4} \kappa_{1} \Delta^{3}+\mathcal{O}\left(\Delta^{4}\right), \Delta+\mathcal{O}\left(\Delta^{2}\right)\right\}, \\
& l_{1} \overrightarrow{\mathbf{t}}_{1}=\left\{-\frac{1}{4} \kappa_{1} \Delta^{3}+\mathcal{O}\left(\Delta^{4}\right), \Delta+\mathcal{O}\left(\Delta^{2}\right)\right\}
\end{aligned}
$$

(cf. (3.2), (4.10), and (4.11)). Similarly, the PH curves of degree 7 match the leading terms of the boundary points $\mathbf{p}_{i}$ (see (4.9)). Note that the unit of the horizontal axes is now $\kappa_{1} \Delta^{3}$ ! Again, three of the four solutions of the first kind lead asymptotically to PH curves with two singularities (cusps) each, corresponding to the simultaneous roots of the components of the hodograph. The remaining solution gives a regular curve; it leads to the best approximation of the original curve among the four solutions.

Remark 4.8. This analysis applies to points with $\kappa_{1} \neq 0$ only, i.e., to nonflat inflection points. A similar analysis could be derived given at inflections and flat points 

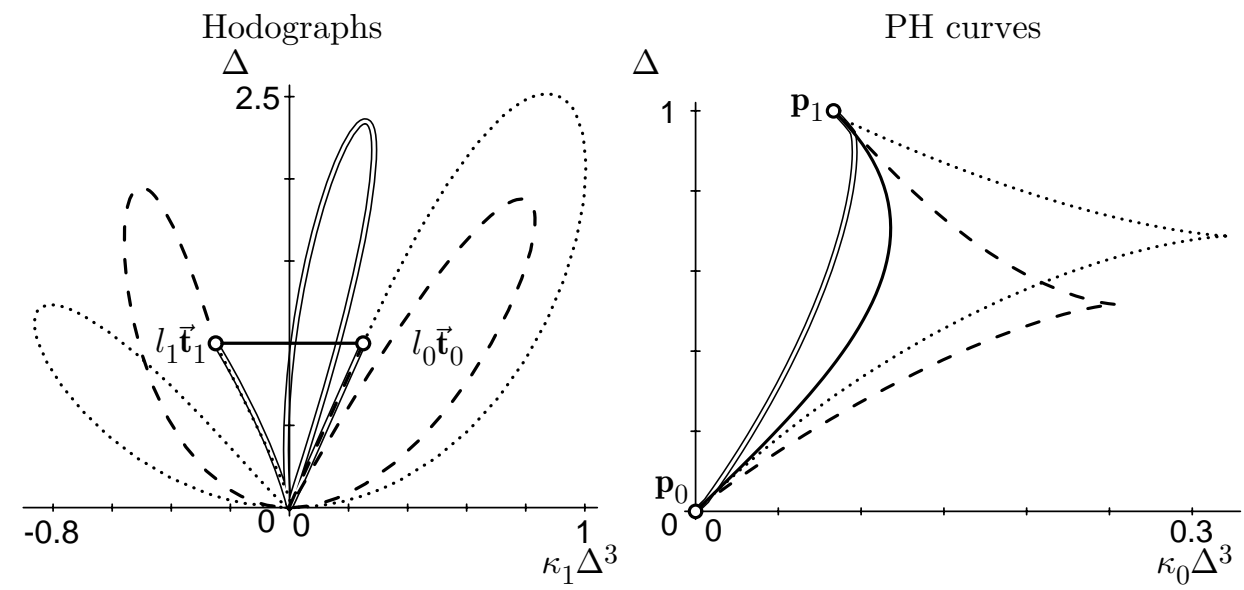

Figure 8. Asymptotic behaviour of the solutions of the first kind (right) $\mathbf{x}(t)$ and of their hodographs $\mathbf{x}^{\prime}(t)$ (left) at an inflection point $\left(\kappa_{0}=0\right)$.

of higher order which are characterized by $\kappa_{0}=\cdots=\kappa_{l}=0, \kappa_{l+1} \neq 0$. So far it is not clear whether Propositions 4.3, 4.5 and 4.7 can be generalized to arbitrarily $l>1$. For instance, if the original curve is "too flat", then one might well imagine that no interpolating PH curve of degree 7 exists. Generating the required Taylor expansions at inflections or flat points of higher order becomes more and more complicated, as terms of higher orders have to be taken into account. At this point we cannot present any further results.

In applications, however, flat points of higher order can often be excluded. For instance, a cubic (spline) curve has points with $\kappa_{0}=\kappa_{1}=0$ only if it (resp. a segment of it) is degenerated into a straight line.

4.5. Approximation order. A general framework for high-accuracy parametric interpolation, and for the discussion of the approximation order in particular, has been developed by Mørken and Scherer 14. With the help of the Taylor expansions from Propositions 4.3 and 4.5 we investigate the approximation order of the $G^{2}\left[C^{1}\right]$ Hermite interpolation scheme with PH curves of degree 7.

Proposition 4.9. If the assumptions of Propositions 4.1 and 4.3 are satisfied (in particular $\left.\kappa_{0} \neq 0\right)$, then the third solution of the first kind has the approximation order 6 .

Proof. In order to prove this result, we generate Taylor expansions of the original curve $\mathbf{p}(T)$, of the third solution of the first kind $\mathbf{x}^{(3)}(t)$, and of the quantities $s$ and $c$ (cf. equations (4.7), (4.13) and (4.10), with residuals in the order of $\mathcal{O}\left(\Delta^{6}\right)$.

Let $U$ be the special orthogonal matrix $U=\left(\begin{array}{cc}-s & c \\ -c & -s\end{array}\right)$. It transforms the solution $\mathbf{x}^{(3)}(t)$ from the adapted system of coordinates (see Figure 3) back into world coordinates, leading to the curve $U \mathbf{x}^{(3)}(t)$. This curve is to be compared with the original curve segment $\mathbf{p}(\tilde{t} \Delta), \tilde{t} \in[0,1]$, of length $\Delta$.

In order to analyze the approximation order of a parametric interpolation scheme, one has to take suitable parameter transformations $\psi$ of the solution into account (cf. [14]). We replace the original parameter $t$ of the third solution of the first kind 
$\mathbf{x}^{(3)}(t)$ with the polynomial

$$
\psi(\tilde{t})=\tilde{t}+\psi_{3}(\tilde{t}) \Delta^{3}+\psi_{4}(\tilde{t}) \Delta^{4},
$$

where

$$
\psi_{3}(\tilde{t})=\frac{\tilde{t}^{2}(1-\tilde{t})^{2}}{\kappa_{0}} C_{3} \quad \text { and } \quad \psi_{4}(t)=\frac{\tilde{t}^{2}(1-\tilde{t})^{2}}{\kappa_{0}^{2}}\left(C_{4,0}+\tilde{t} C_{4,1}\right),
$$

with certain coefficients $C_{3}, C_{4,0}$, and $C_{4,1}$. These coefficients are polynomial expressions of the quantities $\kappa_{i}$ and $r_{i}$ (cf. Proposition 4.1). The polynomials $\psi_{3}$ and $\psi_{4}$ can be found by applying the substitution (4.25) to the solution $U \mathbf{x}^{(3)}(t)$ and comparing the resulting Taylor expansions with the original curve segment $\mathbf{p}(\tilde{t} \Delta)$.

For sufficiently small stepsize $\Delta$, the substitution $\psi(\tilde{t})$ is a bijective transformation of the parameter domain $[0,1]$. Moreover, the parameter transformation $\psi$ preserves the $C^{1}$ boundary data, as

$$
\psi(0)=0, \quad \psi(1)=1, \quad \psi^{\prime}(0)=1, \quad \text { and } \quad \psi^{\prime}(1)=1 .
$$

Using suitable computer algebra tools, it can now be verified that

$$
\left\|\mathbf{p}(\tilde{t} \Delta)-U \mathbf{x}^{(3)}(\psi(\tilde{t}))\right\|=\mathcal{O}\left(\Delta^{6}\right) \text { for all } \tilde{t} \in[0,1] .
$$

This completes the proof.

Without the parameter transformation 4.25), i.e., by choosing the identity $\psi(\tilde{t})=\tilde{t}$, the left-hand side of (4.28) would behave as $\mathcal{O}\left(\Delta^{4}\right)$, which is to be expected for $C^{1}$ Hermite interpolation. A separate discussion is required at inflections of the original curve.

Proposition 4.10. If the assumptions of Propositions 4.1 and 4.5 are satisfied (in particular $\kappa_{0}=0$ and $\kappa_{1} \neq 0$ ), then the third solution of the first kind has the approximation order 5 .

That is, inflections of the original curve lead to a reduction of the approximation order by 1. A similar phenomenon has been observed by de Boor et al. 2] in the case of $G^{2}$ Hermite interpolation with parametric cubic curves.

The proof is omitted; it is analogous to that of the preceding result. The required parameter transformation now takes the form

$$
\psi(\tilde{t})=\tilde{t}+\psi_{2}(\tilde{t}) \Delta^{2}+\psi_{3}(\tilde{t}) \Delta^{3},
$$

where the denominators of the coefficients $\psi_{2}$ and $\psi_{3}$ are $\kappa_{1}$ and $\kappa_{1}^{2}$. Without the parameter transformation, the left-hand side of (4.28) would behave as $\mathcal{O}\left(\Delta^{3}\right)$.

Remark 4.11. In consequence of Remark 4.8 the approximation order at flat points or inflections of higher order $\left(\kappa_{0}=\kappa_{1}=0\right)$ is still open.

4.6. Summary. Summing up, we have the following result.

Theorem 4.12. Consider $G^{2}\left[C^{1}\right]$ Hermite interpolation data which are taken from an analytical curve. If the technical assumptions (4.12), (4.20), and $\left(\kappa_{0}, \kappa_{1}\right) \neq(0,0)$ are satisfied, and provided that the stepsize $\Delta$ is sufficiently small, then the $G^{2}\left[C^{1}\right]$ Hermite interpolation problem with PH curves of degree 7 is solvable, giving eight real solutions. Moreover, at least one regular solution (the third solution of the first kind) exists that matches approximately the shape of the original curve. The approximation order at points with $\kappa_{0} \neq 0$ is 6 ; it is reduced by one at inflections.

As outlined in Remarks 4.6 and 4.8 some of these technical assumptions can probably be eliminated by taking higher order terms of the expansions into account. 


\section{Converting Planar Curves into PH Splines OF DEgree 7}

Based on the results of the preceding sections, we formulate a procedure for approximately converting a given planar curve into a $\mathrm{PH}$ spline curve of degree 7 with any desired accuracy. The original curve is assumed to be globally $C^{2}$, consisting of analytical segments. For instance, this assumption is satisfied by the standard NURBS (NonUniform Rational B-Spline, see [5, 10]) representations. The approximating $\mathrm{PH}$ curves can be computed with the help of the following algorithm.

1) Split the given curve into its analytical segments and apply step 2) to the individual segments. For instance, a given NURB spline curve is to be split into its rational Bézier segments with the help of Boehm's algorithm or knot insertion (see [10]).

2) Take $G^{2}\left[C^{1}\right]$ data from the boundaries of the curve segment and apply the Hermite interpolation procedure (see Table 1) to them. If no suitable solution exists (among the eight potential solutions) or if the accuracy of the approximation is not as good as desired, then split the segment into halves and apply the Hermite interpolation to the new segments. The splitting is iterated until a suitable solution has been found.

In order to benefit directly from the results of the previous section, one might introduce an additional splitting step at the inflections (resp. flat points) of the original curve. For instance, if a cubic (spline) curve $\mathbf{p}(t)$ is given, then these points correspond to roots of the (piecewise) quadratic polynomial $\left[\mathbf{p}^{\prime}, \mathbf{p}^{\prime \prime}\right]$. However, according to our numerical experiences, this additional splitting step is not really necessary and we have therefore abstained from including it in the algorithm.

The second step is guaranteed to be successful after finitely many iterations, as solutions to the Hermite interpolation problem always exist for sufficiently small stepsize (cf. subsection 4.6).

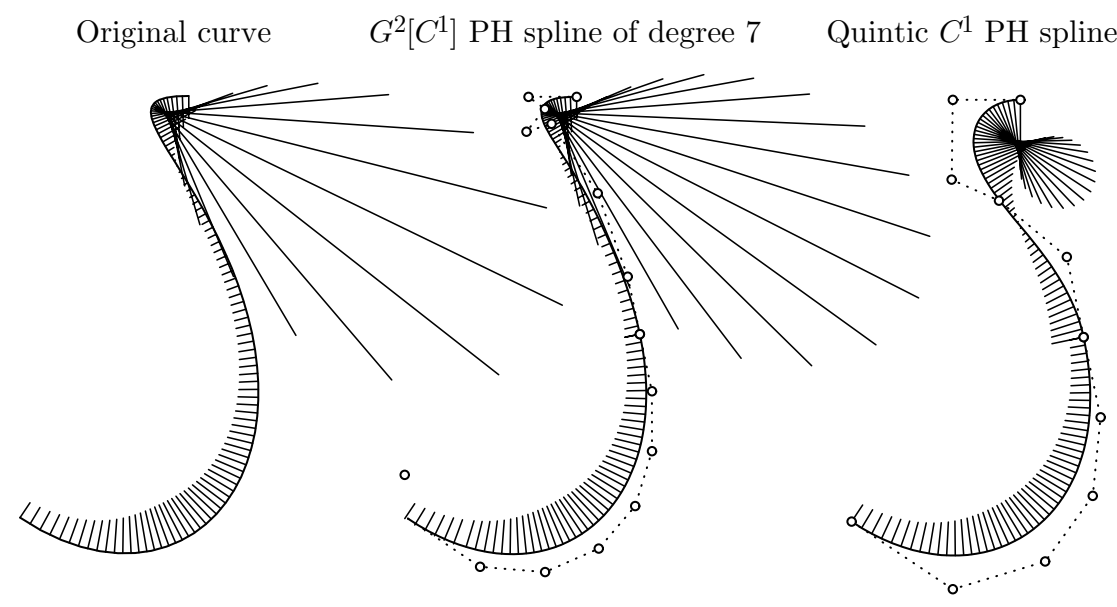

Figure 9. Approximation of a given curve (left) via Hermite interpolation of $G^{2}\left[C^{1}\right]$ data with $2 \mathrm{PH}$ curves of degree 7 (center) and via Hermite interpolation of $C^{1}$ data with $2 \mathrm{PH}$ quintics (right). The scaled curve normals visualize the curvature distribution. 
Theorem 5.1. With the help of the above-described algorithm, any piecewise analytical $C^{2}$ curve can approximately be converted into a polynomial PH spline curve of degree 7 , provided that the technical assumptions of Propositions $4.3,4.5$ and 4.7 are satisfied at all points.

Example 5.2. The original curve is chosen as a planar Bézier curve of degree 4; it is shown on the left-hand side of Figure 9 It is approximated by two PH curves of degree 7 (center), forming a $G^{2}\left[C^{1}\right]$ polynomial PH spline curve. We compare the result with the corresponding results of $C^{1}$ Hermite interpolation by $\mathrm{PH}$ quintics (right) (see 6]). The curvature distribution of the curves is visualized by the scaled curve normals ("porcupines"). Both approximating curves have been drawn along with their control polygons (dotted).

It can clearly be seen that the $\mathrm{PH}$ curves of degree 7 match the original curve quite well. They even reproduce the original curvature distribution. The PH quintics, by contrast, lead to curvature discontinuities, and do not match the original shape as well as desired. Consequently, a bigger number of spline segments would be needed in order to obtain a similar accuracy.

\section{Conclusion}

As the main result of this paper, we have derived a method for constructing curvature continuous polynomial PH spline curves. The construction is based on Hermite interpolation of curvature and first derivative boundary information, and it can be used for approximately converting arbitrary planar curves into polynomial $\mathrm{PH}$ form. As an advantage, the interpolation scheme acts locally, without any need for solving a global system of equations. We have discussed the asymptotic behaviour of the solvability and of the solutions to the Hermite interpolation problem, including the approximation order.

As a matter of further research, we will try to modify the scheme, in order to obtain lower degree interpolants. This may be advantageous in order to reduce the degree of the offset curves.

\section{APPENDIX}

For the convenience of the reader, the components of the symmetric $3 \times 3$-matrices $Q$ and $R$ have been gathered below.

$$
\begin{aligned}
q_{1,1}= & \left(\left(-24 \rho_{0}-20 \rho_{0} \tau_{0}+24 \rho_{1}+20 \tau_{1} \rho_{1}\right) s^{2}+\left(\left(12 \rho_{0}^{2}+20 \tau_{1}^{2}-20 \tau_{0}+20 \tau_{1}\right.\right.\right. \\
& \left.\left.-20 \tau_{0}^{2}-12 \rho_{1}^{2}\right) c-20 \tau_{0}+20 \tau_{1}-20 \tau_{0}^{2}+20 \tau_{1}^{2}\right) s+\left(-10 \tau_{1} \rho_{1}-21 \rho_{1}\right. \\
& \left.+10 \rho_{0} \tau_{0}+21 \rho_{0}-4 \rho_{1} \tau_{0}+4 \tau_{1} \rho_{0}\right) c-10 \tau_{1} \rho_{1}-21 \rho_{1}+10 \rho_{0} \tau_{0}+21 \rho_{0} \\
& \left.-4 \rho_{1} \tau_{0}+4 \tau_{1} \rho_{0}\right) / 70 \\
q_{1,2}= & \left(\left(-24 \rho_{0}+24 \rho_{1}\right) s^{2}+\left(\left(-20 \tau_{0}+20 \tau_{1}\right) c-20 \tau_{0}+20 \tau_{1}\right) s+\left(21 \rho_{0}-21 \rho_{1}\right) c\right. \\
& \left.+21 \rho_{0}-21 \rho_{1}\right) / 140 \\
q_{1,3}= & \left(\left(-24 \rho_{1}-24 \rho_{0}\right) s^{2}+\left(\left(-48-20 \tau_{1}-20 \tau_{0}\right) c-48-20 \tau_{1}-20 \tau_{0}\right) s\right. \\
& \left.+\left(3 \rho_{1}+3 \rho_{0}\right) c+3 \rho_{1}+3 \rho_{0}\right) / 140, \\
q_{2,2}= & 0, \quad q_{2,3}=-12 s(1+c) / 35, \quad q_{3,3}=0,
\end{aligned}
$$


and

$$
\begin{aligned}
r_{1,1}= & \left(\left(12 \rho_{0}^{2}-20 \tau_{1}^{2}-20 \tau_{0}^{2}+12 \rho_{1}^{2}-24-20 \tau_{0}-20 \tau_{1}\right) s^{2}+\left(\left(24 \rho_{1}+20 \rho_{0} \tau_{0}\right.\right.\right. \\
& \left.\left.+24 \rho_{0}+20 \tau_{1} \rho_{1}\right) c+4 \rho_{1} \tau_{0}+21 \rho_{0}+21 \rho_{1}+10 \rho_{0} \tau_{0}+10 \tau_{1} \rho_{1}+4 \tau_{1} \rho_{0}\right) s \\
& +\left(20 \tau_{0}^{2}+2 \tau_{1} \tau_{0}+42+28 \tau_{0}+28 \tau_{1}+20 \tau_{1}^{2}\right) c+42+20 \tau_{0}^{2}+28 \tau_{0}+28 \tau_{1} \\
& \left.+20 \tau_{1}^{2}+2 \tau_{1} \tau_{0}-6 \rho_{0}^{2}-6 \rho_{1}^{2}+9 \rho_{0} \rho_{1}\right) / 70, \\
r_{1,2}= & \left(\left(-12-5 \tau_{1}-5 \tau_{0}\right) s^{2}+\left(\left(6 \rho_{0}+6 \rho_{1}\right) c+21 / 4 \rho_{0}+21 / 4 \rho_{1}\right) s\right. \\
& \left.+\left(21+7 \tau_{1}+7 \tau_{0}\right) c+21+7 \tau_{1}+7 \tau_{0}\right) / 35, \\
r_{1,3}= & \left(\left(-5 \tau_{0}+5 \tau_{1}\right) s^{2}+\left(\left(-6 \rho_{1}+6 \rho_{0}\right) c+3 / 4 \rho_{0}-3 / 4 \rho_{1}\right) s+\left(3 \tau_{0}-3 \tau_{1}\right) c\right. \\
& \left.+3 \tau_{0}-3 \tau_{1}\right) / 35 \\
r_{2,2}= & \left(21+21 c-12 s^{2}\right) / 35, \quad r_{3,3}=\left(3-12 s^{2}+3 c\right) / 35, \quad r_{2,3}=0 .
\end{aligned}
$$

\section{REFERENCES}

1. G. Albrecht and R.T. Farouki, Construction of $C^{2}$ Pythagorean-hodograph interpolating splines by the homotopy method. Adv. Comput. Math. 5 (1996), 417-442. MR 97k:65033

2. C. Boor, K. Höllig and M. Sabin, High accuracy geometric Hermite interpolation. Comput. Aided Geom. Des. 4 (1987), 269-278. MR 90b:65014

3. I.N. Bronshtein and K.A. Semendyayev, Handbook of mathematics, Springer, Berlin, 1997. MR 98a:00003

4. G. Elber, I.-K. Lee, and M.-S. Kim, Comparing Offset Curve Approximation Methods. IEEE Comp. Graphics and Appl. 17 (1998), 62-71.

5. G. Farin, Curves and surfaces for computer aided geometric design, Academic Press, Orlando FL, 1992 (3rd ed.). MR 93k:65016

6. R.T. Farouki and C.A. Neff, Hermite interpolation by Pythagorean hodograph quintics. Math. Comput. 64 (1995), 1589-1609. MR 95m:65025

7. R.T. Farouki and T. Sakkalis, Real rational curves are not 'unit speed', Comput. Aided Geom. Des. 8 (1991), 151-157. MR 92d:65034

8. R.T. Farouki and S. Shah, Real-time CNC interpolators for Pythagorean-hodograph curves. Comput. Aided Geom. Des. 13 (1996), 583-600.

9. R.T. Farouki, Y.-F. Tsai and G.-F. Yuan, Contour machining of free form surfaces with real-time PH curve CNC interpolators. Comput. Aided Geom. Des. 16 (1999), 61-76. CMP 99:04

10. J. Hoschek and D. Lasser, Fundamentals of Computer Aided Geometric Design. AK Peters, Wellesley MA, 1993. MR 94i:65003

11. E. Kreyszig, Differential geometry, Oxford University Press, London, 1964. MR 92d:53001

12. K.K. Kubota, Pythagorean triples in unique factorization domains. Am. Math. Monthly 79 (1972), 503-505. MR 45:6742

13. D.S. Meek and D.J. Walton, Geometric Hermite interpolation with Tschirnhausen cubics. J. Comput. Appl. Math. 81 (1997), 299-309. MR 98b:65011

14. K. Mørken and K. Scherer, A general framework for high-accuracy parametric interpolation. Math. Comput. 66 (1997), 237-260. MR 97e:65026

15. J. Peters and U. Reif, The 42 equivalence classes of quadratic surfaces in affine $n$-space. Comput. Aided Geom. Des. 15 (1998), 459-473. MR 99e:14603

16. H. Pottmann, Curve design with rational Pythagorean-hodograph curves. Adv. Comput. Math. 3 (1995), 147-170. MR 96h:65024

17. D.J. Walton and D.S. Meek, $G^{2}$ curves composed of planar cubic and Pythagorean hodograph quintic spirals. Comput. Aided Geom. Des. 15 (1998), 547-566. MR 99c:65040

Institute of Analysis and Computational Mathematics, Johannes Kepler University Linz, Altenberger Str. 69, 4040 Linz, Austria

E-mail address: Bert.Juettler@jk.uni-linz.ac.at 